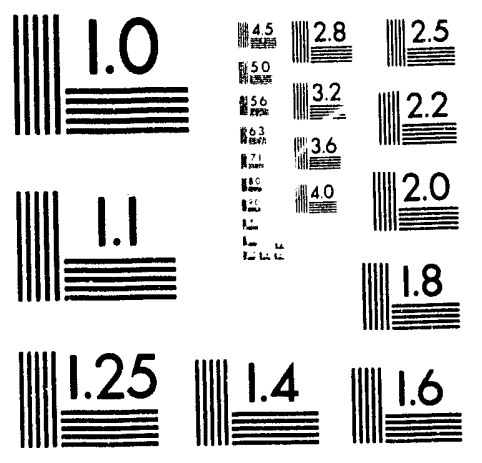



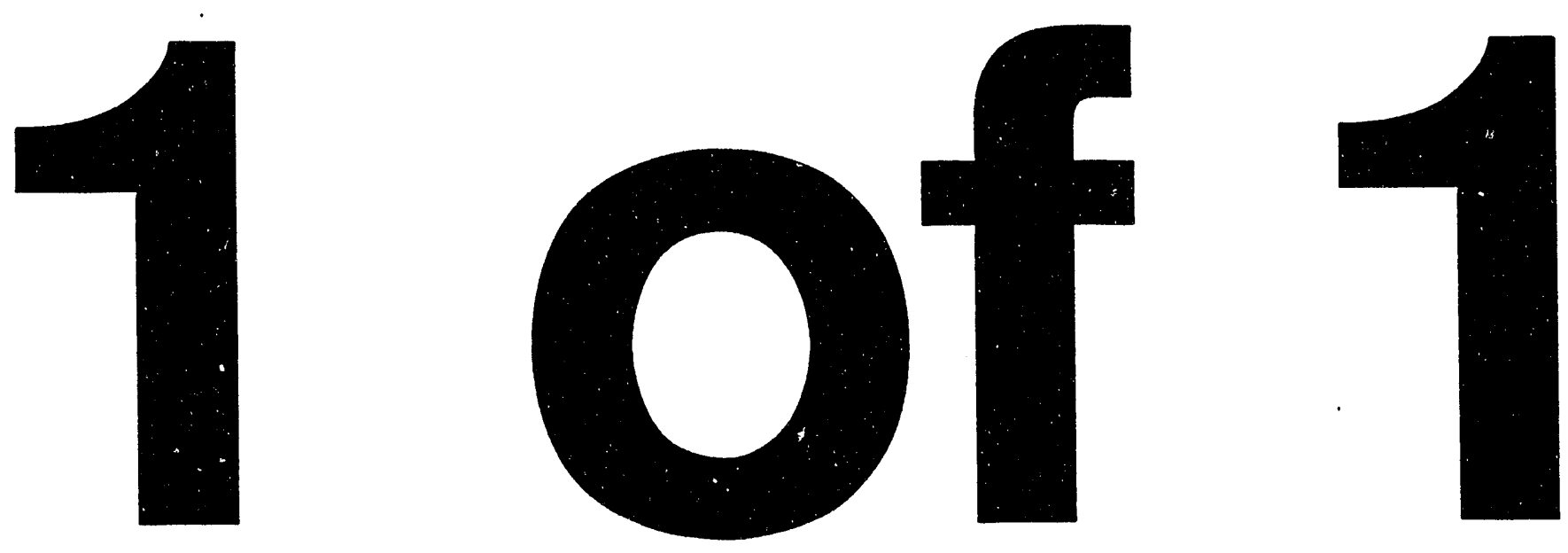


\title{
PVDF GAUGE CHARACTERIZATION OF HYPERVELOCITY-IMPACT-GENERATED DEBRIS CLOUDS
}

\author{
M.B. Boslough, L.C. Chhabildas, W.D. Reinhart, C.A. Hall, J.M. Miller, R. Hickman \\ Experimental Inpact Physics Department 1433 \\ Saudia National Laboratories \\ Albuquerque, New Mexico, USA 87185-5800
}

\author{
S.A. Mullin, and D.L. Littlefield \\ Southwest Research Institute \\ San Antonio, Texas, USA 78228-0510
}

We have used PVDF gauges to determine time-resolved stresses resulting from interaction between hypervelocity-impactgenerated debris clouds and various target gauge blocks. Debris clouds were generated from three different impact configurations: 1) steel spheres impacting steel bumper sheets at 4.5 to $6.0 \mathrm{~km} / \mathrm{s}, 2$ ) aluminum inhibited shaped-charge jets impacting aluminum bumper sheets at $11.4 \mathrm{~km} / \mathrm{s}$, an' 3 ) titanium disks impacting titanium bumper sheets at 7.6 to $10.1 \mathrm{~km} / \mathrm{s}$. Additional data were collected from the various experiments using flash X-ray radiography, pulsed laser photography, impact flash measurements, time-resolved strain gauge measurements, and velocity interferometry (VISAR). Data from these various techniques are in general agreement with one another and with hydrocode predictions, and provide a quantitative and comprehensive picture of impact-generated debris clouds.

\section{INTRODUCTION}

There have been many experimental characterization studies of hypervelocity debris clouds using a number of diagnostic methods [e.g. 1-3]. Techniques have included recovery of witness plates, collection of flash X-ray images, and fast framing photography. More recently, pulsed-laser, open-shutter photography and holography have been used in an effort to provide higher-resolution data on spatial and size distributions of particles.

The purpose of the present work is to continue to develop quantitative methods for the characterization of hypervelocity-impact generated debris. Polyvinyidene difluoride (PVDF) gauges have been shown to be reliable and convenient for collecting time-resolved stress data in a number of applications, particularly those involving very fast loading rates under uniaxial strain [4]. By embedding them inside a solid material placed in the path of a debris cloud moving at hypervelocity, information about the structure of the debris cloud can be inferred from the measured stress histories. In addition, hydrocode simulations of impact-generated debris clouds can include their effect on a solid gauge block. By comparing measured stress histories to those predicted by the code, the experiments can be used to validate hydrodynamic codes in a quantitative way.

\section{EXPERIMENTAL METHODS}

Three sets of experiments were performed using three
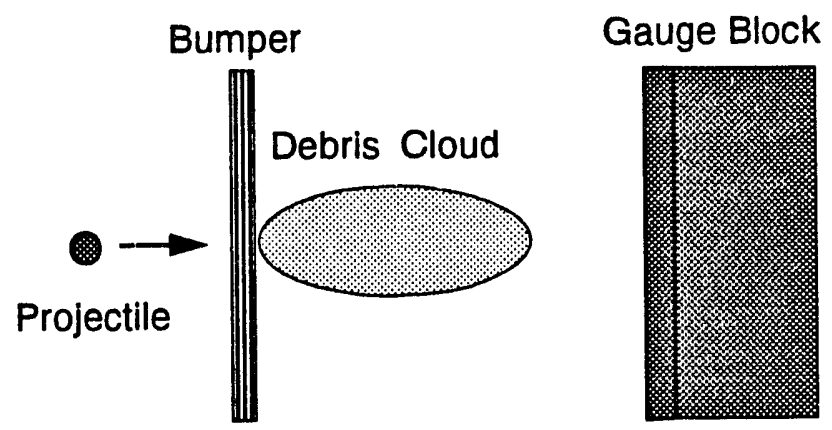

Figure 1. General Experimental Configuration

different launch systems. The general configuration is shown in Fig. 1. A bumper plate is impacted by a hypervelocity projectile, generating a debris cloud which subsequently impacts upon a solid gauge block. The hypervelocity projectiles included steel spheres, aluminum shaped charge jets, and titanium disks.

\section{PVDF Gauges}

There is more than one type of PVDF gauge, and there are several modes in which they can be operated. For these experiments we made use of both Bauer [e.g., 4] and Dynasen [5] gauges. For applications in which extremely fast (ns) response is required, gauges are typically operated in current mode. A precision current-viewing resister (CVR) is placed in parallel with the gauge at the target, and the measured current is mathematically integrated to yield charge, from which stress can be determined.

\section{MASTER}




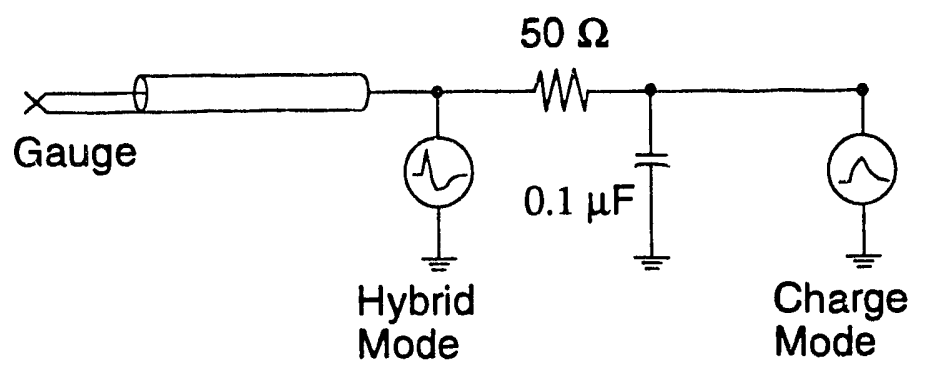

Figure 2. Circuit diagram for PVDF gauges

For the present experiments, in which stress rise times are hundreds of nanoseconds to microseconds, it was found that charge-mode recording was more useful. In charge mode, the signal from the gauge is input into a lowinductance harjware charge integrator (HCI) which contains a $0.1 \mu \mathrm{F}$ capacitor. The charge is recorded directly as a voltage drop across the capacitor (Figure 2). By measuring the voltage difference across the top side of the $\mathrm{HCI}$ (looking across a $50 \mathrm{ohm}$ resistor), an effective currentmode measurement can be made simultaneuusly. Because of the back voltage due to the charge on the capacitor, this measurement is the sum of voltages due to charge and current, and is therefore referred to as "hybrid mode" in this paper.

There are two methods of determining the equivalent current mode signal from hybrid mode: 1) if a good charge mode record was made using the same time base, it can be subtracted directly, or 2) the integrated hybrid mode record can be subtracted (with appropriate constants from calibration) from the original record. This procedure is repeated iteratively until convergence. The advantage in combined hybrid/charge mode recording is that it is very forgiving of sensitivity settings, which is important for experiments such as these, in which peak stresses and risetimes are difficult to predict.

Most of the development and application of PVDF gauges has been limited to environments of uniaxial strain conditions generated by planar loading experiments. In the present applications, the debris clouds have a more complicated geometry and contain discrete solid fragments, liquid droplets, and vapor, generally giving rise to nonuniaxial loading conditions within the target. The fact that thin-film PVDF gauges respond to lateral strain as well as normal stress needs to be considered. As will be seen in the following section, this was done on a case-by-case basis.

\section{RESULTS AND DISCUSSION}

Because of the large number of experiments carried out using a variety of geometries, loading conditions, and materials, only selected shots from each of the three series will be discussed.

\section{Steel Spheres onto Steel Plates}

In the first series, steel spheres were launched with the Terminal Ballistics Facility (TBF) gun at Sandia's STAR Facility. They impacted onto steel plates, and the resulting debris clouds impacted PMMA disks containing four embedded PVDF gauges. Additional data were collected using flash $\mathrm{X}$-ray radiography, pulsed-laser photography, and impact flash measurements for timing.

\section{Experiment XT-9}

Shown in Figure 3 are a pulsed laser photograph and a flash $x$-ray image of a debris cloud generated by the impact of a $6.35 \mathrm{~mm}$ sphere at $4.50 \mathrm{~km} / \mathrm{s}$. These two images were obtained simultaneously, approximately $23 \mu \mathrm{s}$ after impact on the bumper plate. The time of impact was precisely determined by measuring the light emission (impact flash) with a photomultiplier tube. By measuring the positions of the debris front on the visible and $\mathrm{X}$-ray images, the debris front velocity was determined to be 4.28 and $4.31 \mathrm{~km} / \mathrm{s}$, respectively. This velocity was independently confirmed with the time-of-arrival of the first compression wave to the PVDF gauges, which yielded a debris velocity of $4.29 \mathrm{~km} / \mathrm{s}$.

\section{Experiment XT-5}

This experiment was identical to XT-9 except that the impactor was smaller (4.76 $\mathrm{mm}$ diameter). The PMMA gauge block contained four PVDF gauges in an array, with one at the center and the other three approximately equally spaced on a $25.4 \mathrm{~mm}$ radius. The projectile trajectory was about $18 \mathrm{~mm}$ below and $10 \mathrm{~mm}$ to the left of the center line of the target, so that each gauge was a different distance from the axis of the debris cloud. The measured stress histories from each gauge are presented in Figure 4. The time of arrival of the stress peak at each gauge increases with that gauge's distance from the center axis of the debris cloud, as can be seen in the inset plot in Figure 4.
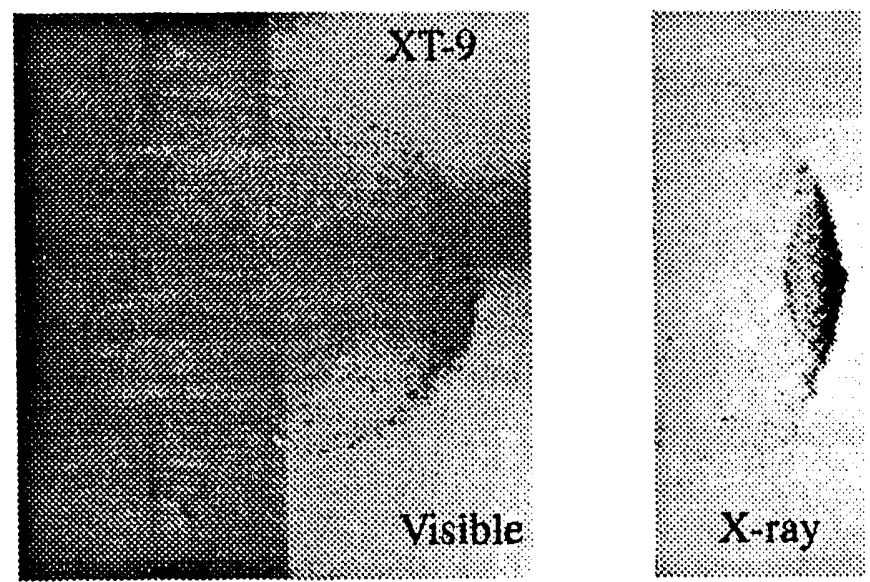

Figure 3. Visible and X-ray images of steel debris 
Integrating the stress history with respect to time yields an impulse history, which contains information about the structure of the debris cloud. A set of impulse histories is displayed in Figure 5. In the inset, the impulse at the time peak pressure is achieved at each gauge is displayed as a function of that gauges radial distance from the axis of the debris cloud. This plot demonstrates that the momentum density is approximately symmetric within the debris cloud, consistent with the images in Figure 3 . In this set of experiments, it was assumed a priori that the contribution from lateral strain to the gauge output was not important for the first one or two microseconds.

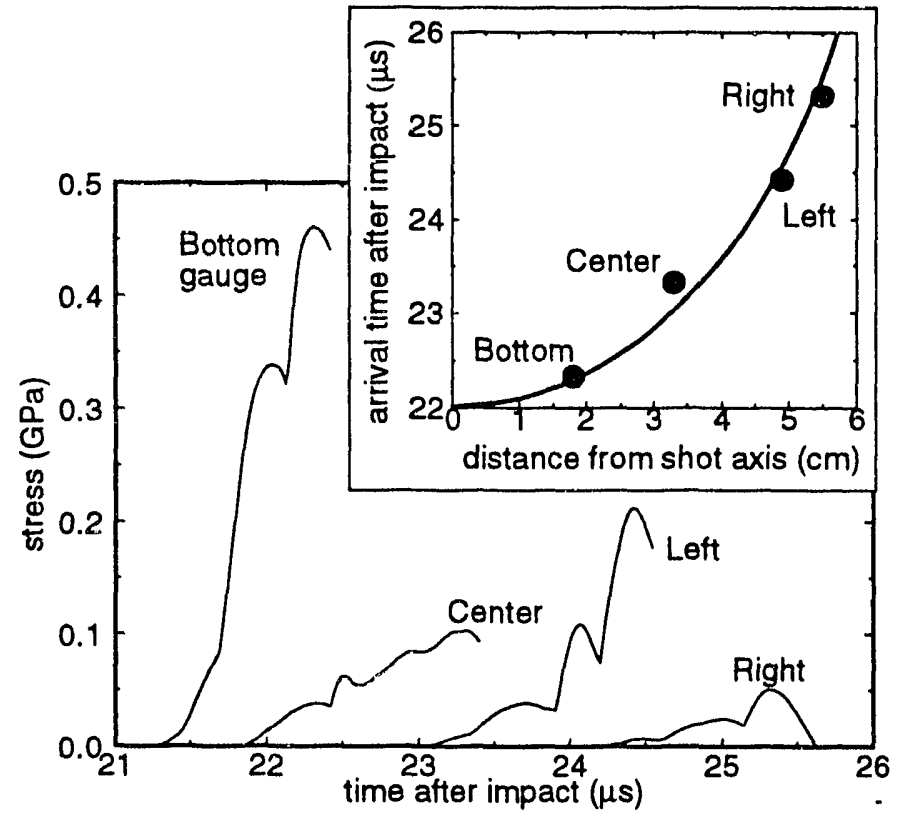

Figure 4. Four stress histories from XT-5

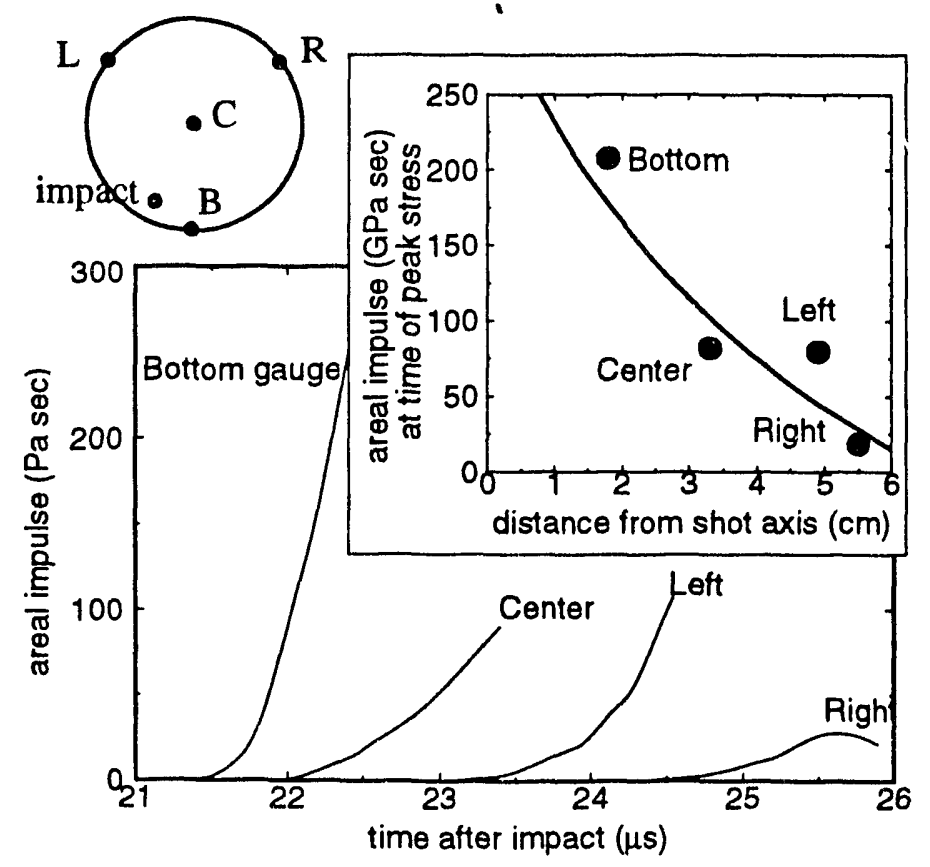

Figure 5. Four impulse histories from XT-5

\section{Aluminum Jets onto Aluminum Plates}

Experiments using aluminum inhibited shaped charge jets were performed at Southwest Research Institute. The 4.6 mm diameter $(\mathrm{L} / \mathrm{D}=3)$ aluminum projectiles were launched at speeds of $11.4 \mathrm{~km} / \mathrm{s}$, and impacted onto a $1.27 \mathrm{~mm}$-thick aluminum sheet. An aluminum gauge block was placed $200 \mathrm{~mm}$ away from the bumper, and had two Bauer PVDF gauges embedded $30 \mathrm{~mm}$ behind its front surface to measure the stress profile generated by the impact of the aluminum debris cloud. In addition, orthogonal flash $\mathrm{X}$-ray images were obtained of both the projectile and the debris cloud.

\section{Experiment T-37}

In preparation for these experiments a series of CTH hydrocode simulations was performed for various impact/ target configurations. One purpose of these calculations was to provide an estimate of the expected shock pressures and risetimes. A second goal was to determine the optimal depth at which the gauges should be embedded in the target, so they would be deep enough that lateral strain would be small, but sufficiently shallow that they would still be sensitive to the structure of the debris cloud. Based on the results of the simulations we chose to embed the gauges $30 \mathrm{~mm}$ from the front surface of the target.

Figure 6 displays $\mathrm{X}$-ray images of the shaped-charge jet prior to impact, and the resulting debris cloud. As can be seen, the projectile is not a perfect right circular cylinder, and the spatial distribution of the debris departs from the ideal axial symmetry of the CTH simulation. Nevertheless, a comparison of the PVDF gauge output to the predicted stress history for a $11.1 \mathrm{~km} / \mathrm{s}$, axially symmetric impact shows remarkable agreement (Figure 7). The center of the crater in the recovered target was about $15 \mathrm{~mm}$ away from the gauge position, so the stress tracer at that point was chosen for comparison. While the exceptional agreement may be somewhat fortuitous, this comparison underscores the fact that PVDF gauges can provide a strong quantitative tool for hypervelocity impact code validation.
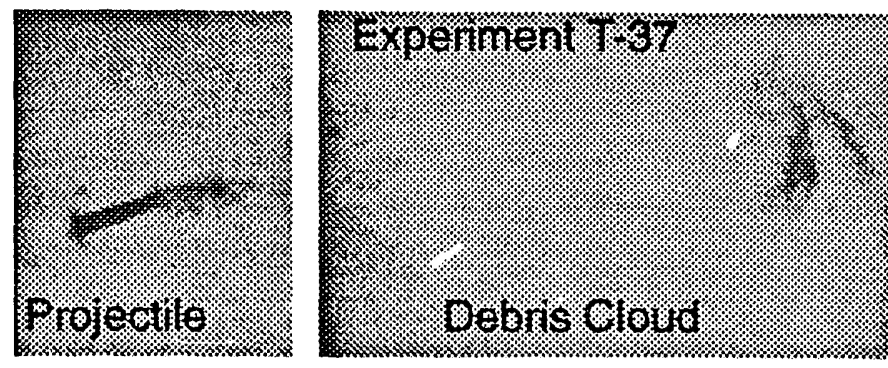

Figure 6. X-ray images of aluminum jet and debris 


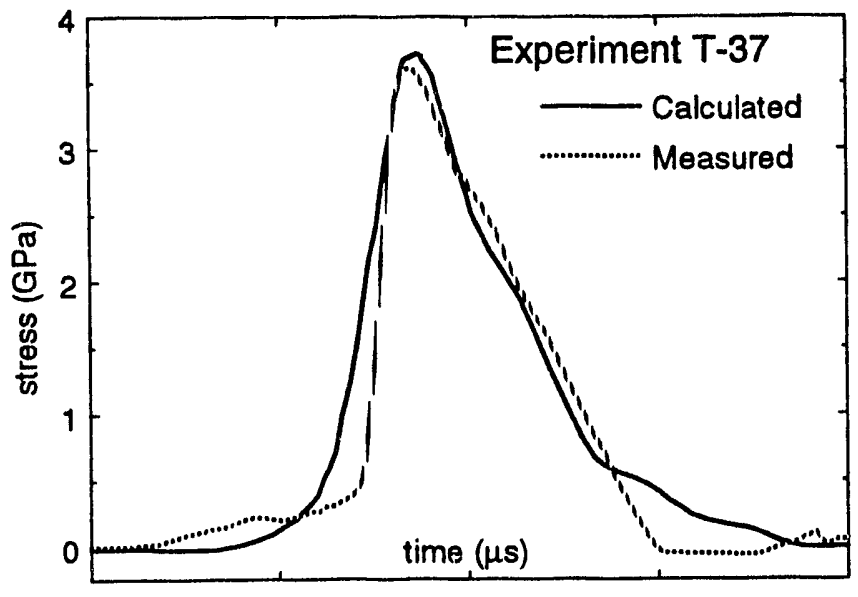

Figure 7. Calculated and measured stress

\section{Titanium Disks onto Titanium Plates}

These experiments made use of Sandia's Hypervelocity Launcher (HVL). Titanium disks $17 \mathrm{~mm}$ in diameter and about $0.5 \mathrm{~mm}$ thick were launched to speeds of 7.6 to $10.1 \mathrm{~km} / \mathrm{s}$. The debris cloud generated upon impact with a titanium bumper plate was allowed to impact a PMMA gauge block mounted $305 \mathrm{~mm}$ away. This target contained two PVDF gauges, each overlying two strain gauges for a time-resolved measurement of lateral strain at the gauge locations. A simultaneous VISAR measurement was made from a location directly behind one of the PVDF gauges, separated by a thin (0.7-mm thick) PMMA buffer. The strain measurements and VISAR measurements were made to determine the magnitude of strain compensation required. Flash X-rays were also obtained of the flyer and of the debris cloud after impact.

\section{Experiment $\mathrm{HTI} \cdot 7$}

Figure 8 shows $x$-ray images of a titanium disk about to impact at $7.6 \mathrm{~km} / \mathrm{s}$, and the resulting debris cloud. The disk was damaged during launch, and is rotated. In Figure 9, preliminary VISAR, PVDF, and strain data are plotted on the same time scale. This test was completed very recently, and has not yet been fully analyzed; however, it is noteworthy that the lateral strain is significant. The stress at the gauge position will be determined independently from VISAR data and from strain-compensated PVDF data.

\section{SUMMARY}

PVDF gauges provide a convenient quantitative means of characterizing debris clouds generated by hypervelocity impacts, with compensation for strain required in some cases. We anticipate that they will become an increasinglyused tool for the experimental validation of hydrodynamic code simulations of hypervelocity impact events.
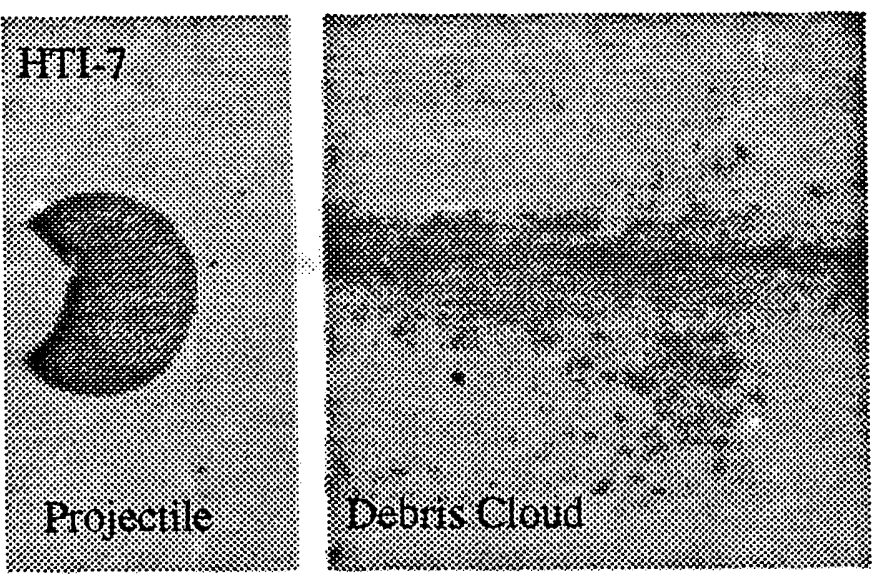

Figure 8. X-ray images of titanium disk and debris

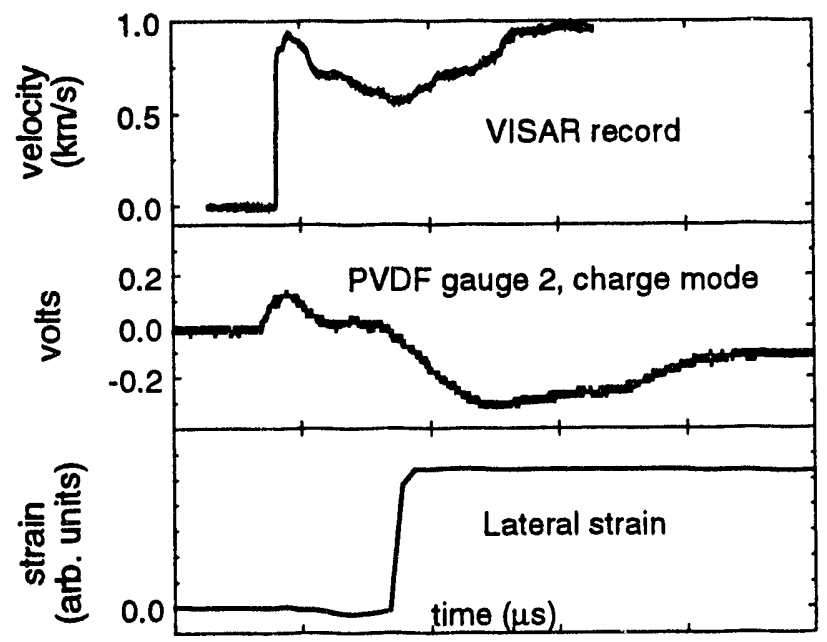

Figure 9. Preliminary data on HTI-7.

\section{REFERENCES}

[1] M. Boslough et al., Intl. J. Impact. Engng., 14, (1993). [2] L.C. Chhabildas et al., "Debris Cloud Characterization at Impact Velocities over 5 to $11 \mathrm{~km} / \mathrm{s}$." this volume.

[3] C.H. Konrad et al., "Dependence of Debris Cloud Formation on Projectile Shape," this volume.

[4] F. Bauer, et al., in Shock Compression of Condensed Matter 1991, ed. by S.C. Schmidt et al., p. 887, (1992).

[5] J.A. Charest and C.S. Lynch, in Shock Compression of Condensed Matter 1991 , ed. by S.C. Schmidt et al., p. 897 , (1992).

\section{ACKNOWLEDGMENTS}

M.U. Anderson, D.E. Wackerbarth, D.A. Crawford, D.K. Johnson, J.A. Charest, and C.S. Lynch provided expert technical advice and help. This work was performed at Sandia National Laboratories supported by the U.S. Department of Energy under contract DE-AC04$76 \mathrm{DP} 00789$. 

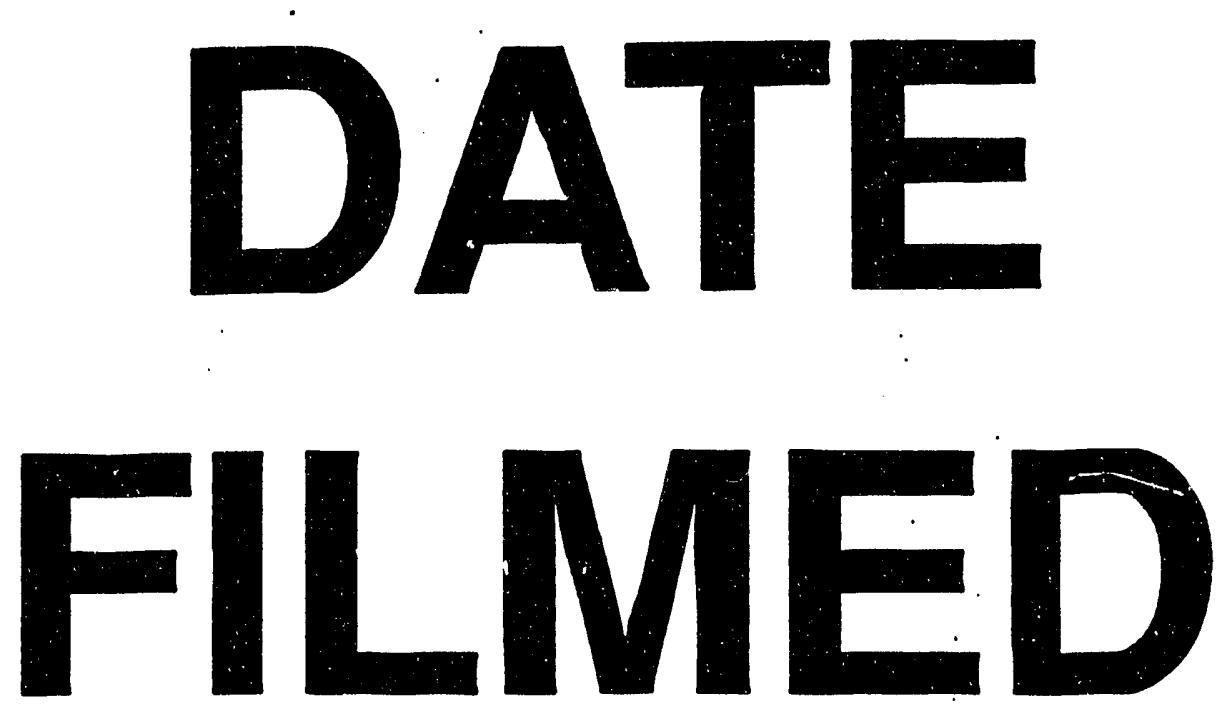

$11 / 4 / 93$
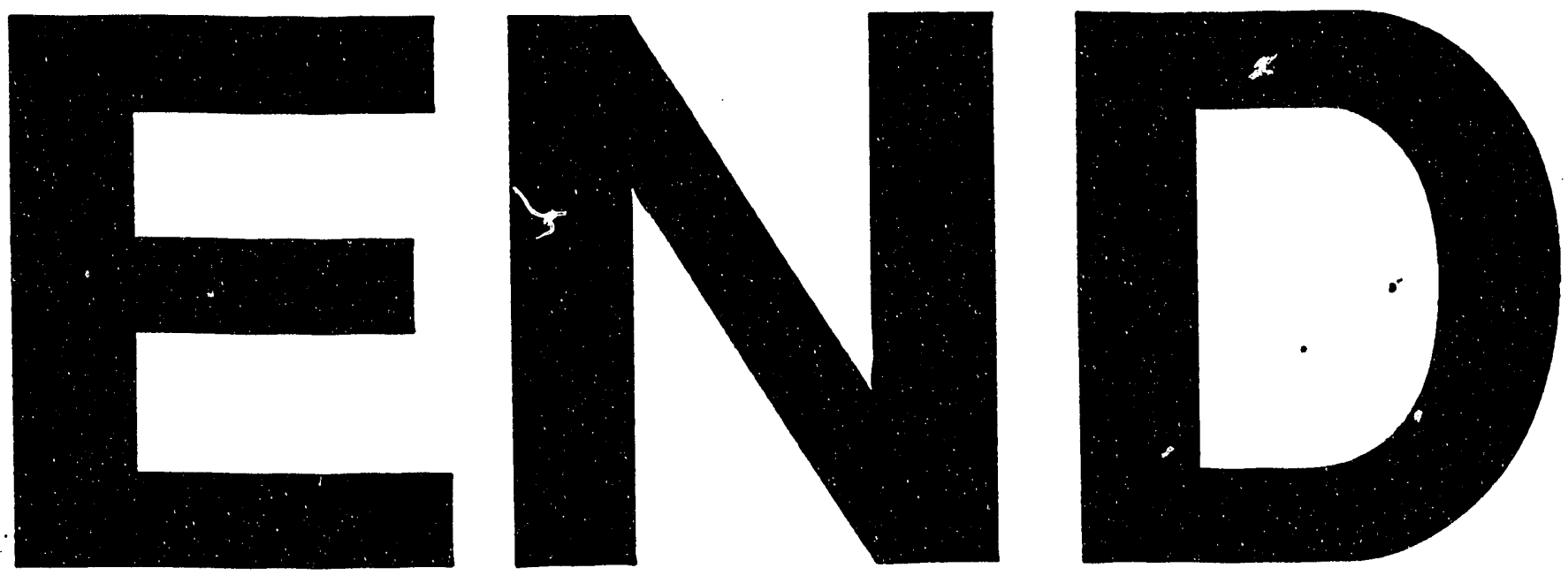
